Braz J Med Biol Res, January 2012, Volume 45(1) 38-42

doi: 10.1590/S0100-879X2011007500155

\section{Postural balance in patients with social anxiety disorder}

M.N. Levitan, J.A. Crippa, L.M. Bruno, D.L. Pastore, R.C. Freire, K.C. Arrais, J.E. Hallak and A.E. Nardi

The Brazilian Journal of Medical and Biological Research is partially financed by

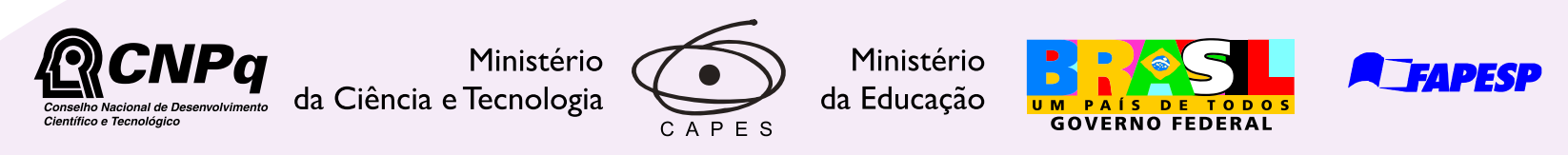

Institutional Sponsors
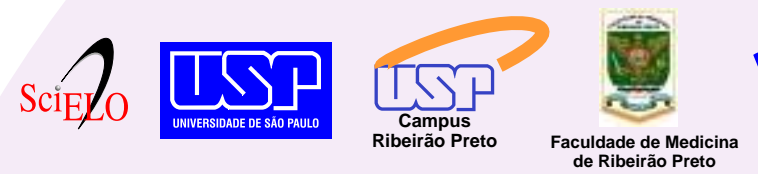

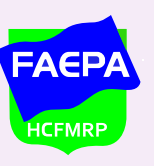

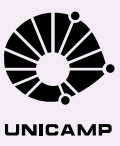

SHIMADZU

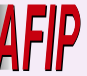

Associaçä Fundo del Incentivo
a Pesquisa

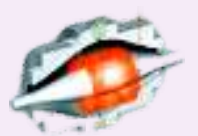
UNICAMP

ore High - Performance MS Orbitrap Technology In Proteomics \& Metabolomics analice Thermo 


\title{
Postural balance in patients with social anxiety disorder
}

\author{
M.N. Levitan ${ }^{1,2}$, J.A. Crippa 2,3 , L.M. Bruno ${ }^{1,2,4}$, D.L. Pastore ${ }^{1,2,4}$, \\ R.C. Freire ${ }^{1,2}$, K.C. Arrais ${ }^{3}$, J.E. Hallak ${ }^{2,3}$ and A.E. Nardi ${ }^{1,2}$ \\ ${ }^{1}$ Laboratório do Pânico e Respiração, Instituto de Psiquiatria, \\ Universidade Federal do Rio de Janeiro, Rio de Janeiro, RJ, Brasil \\ 2 Instituto Nacional de Ciência e Tecnologia Translacional em Medicina, Rio de Janeiro, RJ, Brasil \\ ${ }^{3}$ Departamento de Neurociências e Ciências do Comportamento, Hospital das Clínicas, \\ Faculdade de Medicina de Ribeirão Preto, Universidade de São Paulo, Ribeirão Preto, SP, Brasil \\ ${ }^{4}$ Faculdade de Medicina, Centro de Ciências da Saúde, Universidade Federal do Rio de Janeiro,
}

Rio de Janeiro, RJ, Brasil

\begin{abstract}
Body stability is controlled by the postural system and can be affected by fear and anxiety. Few studies have addressed freezing posture in psychiatric disorders. The purpose of the present study was to assess posturographic behavior in 30 patients with social anxiety disorder (SAD) and 35 without SAD during presentation of blocks of pictures with different valences. Neutral images consisted of objects taken from a catalog of pictures, negative images were mutilation pictures and anxiogenic images were related to situations regarding SAD fears. While participants were standing on a force platform, similar to a balance, displacement of the center of pressure in the mediolateral and anteroposterior directions was measured. We found that the SAD group exhibited a lower sway area and a lower velocity of sway throughout the experiment independent of the visual stimuli, in which the phobic pictures, a stimulus associated with a defense response, were unable to evoke a significantly more rigid posture than the others. We hypothesize that patients with SAD when entering in a situation of exposure, from the moment the pictures are presented, tend to move less than controls, remaining this way until the experiment ends. This discrete body manifestation can provide additional data to the characterization of SAD and its differentiation from other anxiety disorders, especially in situations regarding facing fear.
\end{abstract}

Key words: Social anxiety disorder; Postural control; Freezing; Stabilometry; Anxiety; Social exposure

\section{Introduction}

Postural sway during quiet standing reflects the interplay between the destabilizing forces acting on the body, such as gravity and external environment, and the reaction of the postural control system to prevent the loss of balance. Balance impairments caused by central nervous malfunction are reflected in altered postural sway, such as observed in Parkinson's disease (1). The best measures of normal and altered postural sway are still being developed (2).

Few studies have investigated the effect of muscle stiffness on sway amplitude. Animal models suggest that different defensive patterns might be associated with specific anxiety disorder (3). For example, threatening cues activate defensive neural circuits in animals that facilitate the processing of the threat context, thus preparing the organism for overt defensive behavior (4). For many investigators, the freezing response is a behavior in the defensive cascade where the postural sway area is reduced and the animal abruptly stops, monitors the source of danger, and prepares for fight or flight (5-7).

The rigid posture present in the defense behavior has received relatively little scientific attention in humans. Since some studies on freezing posture and psychiatric disorders such as specific phobia, post-traumatic stress disorder (PTSD) and panic disorder (PD) have been performed, but no studies with social anxiety disorder (SAD) have been conducted so far, the importance of our study is to integrate the investigation regarding the psychobiology of anxiety disorders, performing a similar investigation with SAD.

Studies have found that individuals with fear of heights showed a more increased sway than healthy individuals

Correspondence: M.N. Levitan, Laboratório do Pânico e Respiração, Universidade Federal do Rio de Janeiro, R. Visconde de Pirajá, 407/702, 22410-003 Rio de Janeiro, RJ, Brasil. Fax: +55-21-2523-6839. E-mail: milevitan@hotmail.com

Received February 15, 2011. Accepted October 31, 2011. Available online November 18, 2011. Published January $16,2012$. 
when exposed to visual flow stimulation studies, suggesting that a high state of anxiety increased postural sway in healthy individuals, with an increment of path length in the anteroposterior axis $(8,9)$. On the other hand, studies using fearful situations, such as viewing images of mutilation, showed a decrease in the amplitude of postural sway and an increase in the mean power frequency, where fear was pointed out as being involved in different neuroanatomic substrates and psychological outcomes (8).

These results were not replicated by Lopes et al. (10), who presented pictures with different valences (neutral, mutilation, and anxiogenic) to 29 PD patients and found a significantly reduced body sway area, a reduced mean power frequency thorough the experiment and a negative correlation between anticipatory anxiety and mean sway area when compared to control participants.

Regarding PTSD, a study examined the prevalence of tonic immobilization in 35 rape survivors on the basis of self-reported degree of paralysis/freezing experienced during the rape. Results showed that $37 \%$ of participants experienced complete immobility during their sexual assaults (11).

Therefore, the aim of the present study was to analyze the postural control response in SAD patients and healthy controls viewing affective pictures (neutral, phobic and negative) with a stabilometric method. The present study can help us to identify how subjects react to safety and danger stimuli, providing new perspectives and investigations about the etiological hypothesis of SAD and other anxiety disorders. To our knowledge, no previous studies have examined the postural behavior of SAD subjects.

Based on previous research $(10,12)$, we expected that patients with SAD would demonstrate reduced body sway when exposed to the phobic pictures compared to controls, but a similar reactivity to control participants during fear contexts in which defensive mobilization is normal and adaptive, such as viewing negative pictures (13).

\section{Material and Methods}

Thirty generalized SAD outpatients (18 women and 12 men) and 35 healthy control subjects (22 women and 13 men) participated in this study. All subjects were recruited from the sample of an epidemiologic survey in which 2319 university students completed self-assessment diagnostic instruments $(14,15)$. Of these, 474 were selected and assigned to two groups: i) 237 individuals with a probable SAD diagnosis who, when screened with the validated reduced version of the Social Phobia Inventory (MINI-SPIN) to Portuguese (16), had a score of at least six points in the three items, and ii) 237 volunteers with similar sociodemographic characteristics who scored zero in the three items of the MINI-SPIN.

All 474 subjects were then contacted by telephone and asked to complete the semantically adapted Portuguese version (17) of the anxiety mode of the Structured Clinical Interview for DSM-IV, clinical version (SCID-CV) (18). Following the completion of the interview, the subjects were again divided into two groups: i) an experimental group consisting of individuals with SAD, and ii) a control group consisting of healthy individuals. Finally, the full version of the SCID-CV was used for diagnostic confirmation and exclusion of comorbid conditions (19). The Subjective Units of Disturbance Scale (20) was administered to measure anxiety before and after the experiment.

Subjects were excluded if they presented other psychiatric disorders, except for a previous depressive episode, since this is a frequently comorbid condition with SAD (21), in which medication could be used. Additionally, we excluded individuals under treatment with psychotropic medication or taking psychoactive substances for other psychiatric disorders, and subjects who presented a general medical condition or who had received previous treatment for SAD (either pharmacological treatment or psychotherapy). We excluded subjects based on the following criteria: cardiovascular, respiratory, vestibular or orthopedic diseases, hypertension, pregnancy, and epilepsy. The research protocol was approved by the Ethics Committee of the Instituto de Psiquiatria (Universidade Federal do Rio de Janeiro) and Hospital das Clínicas (Faculdade de Medicina, USP) and all volunteers gave written informed consent to participate.

\section{Subjects and procedure}

Subjects were instructed to stand barefoot on a force platform and to remain quiet with their feet together and arms relaxed along the trunk while looking at a screen. A mobile AccuSway Plus force platform created by Advanced Mechanical Technology, Inc. (USA) measured postural sway.

\section{Visual stimuli}

The emotional visual stimuli consisted of 48 pictures (16 neutral, 16 of social threat, and 16 of aversive reaction). The negative block consisted of mutilation pictures selected from the International Affective Picture System (22). Objects and utensils, also drawn from this catalog, were used as a neutral category. The anxiogenic pictures consisted of distressing situations for the SAD patients, such as social presentations, get-togethers and interview scenarios. The anxiogenic pictures were evaluated by an independent sample of patients with SAD from a group of 30 pictures regarding social encounters, rated from 0-10 according to the degree of discomfort they caused in the evaluators. The 16 pictures with the highest scores were included in the experiment.

\section{Experimental design}

The experimental protocol consisted of the blocked presentation of 16 pictures of each category in a permanent order as neutral-anxiogenic-negative. The negative block 
was the last to appear since the reaction of horror to physical threat pictures can interfere with the rest of the experiment. Pictures were presented for $3 \mathrm{~s}$ each (48 s per block), and each block was preceded and followed by a stationary gray screen for $48 \mathrm{~s}$. The session on the platform lasted $5 \mathrm{~min}$ and $36 \mathrm{~s}$ without interruption. Since there are no other studies with psychiatric disorders, the length of the experiment was based on Lopes et al. (10) who successfully used this design for PD.

\section{Physiological response measurement}

Stabilometric signals were sampled at $50 \mathrm{~Hz}$ using an anti-aliasing filter with a cut-off frequency of $5 \mathrm{~Hz}$. Based on the displacement of the center of pressure in the mediolateral and anteroposterior directions, we calculated standard deviation (SD), mean velocity, mean power frequency, and elliptical sway area.

\section{Statistical analysis}

The posturographic data were evaluated by two-way fixed-effects ANOVA. The factors analyzed were group (SAD and control) and valence (neutral, anxiogenic, and negative). A t-test was used to compare pre- and post-experiment anxiety levels between groups. Chisquare tests were used to compare demographic data. The Pearson correlation coefficient was used to test correlations between variables.

\section{Results}

\section{Posturography}

From the total sample of 65 subjects, we excluded 2 persons from the SAD group and 1 person from the control group because they would not stand still on the platform. In addition, 3 subjects from the SAD group and 2 from the control group showed stabilometric parameters of more than $3 \mathrm{SD}$ from the respective group average and were excluded from the analysis.

\section{Descriptive data/independent variables}

There were no statistical differences in demographic data between groups. The total sample consisted of $66.1 \%$ women $(P=0.159)$ with a mean age of $26 \pm 4.8$ SD $(P=0.541)$. Furthermore, $73.2 \%$ of the sample was single $(P=0.275)$ and $60.7 \%$ had at least complete high school $(P=0.211)$.

There was a significant difference between groups in the use of antidepressants (6.7 and $32 \%$ for controls and patients, respectively; $P \leq 0.05)$. There was no significant difference between group means in the state of anxiety before the experiment, as evaluated by the Subjective Units of Disturbance Scale $(4.3 \pm 2.5 \mathrm{SD}, \mathrm{P}=0.137)$, but phobic subjects ended the experiment more anxious (3.2 and 4.5; $\mathrm{P} \leq 0.05$ ) than controls.
Table 1. Posturographic parameters of the groups.

\begin{tabular}{lcc}
\hline Posturographic parameters & Controls & Patients \\
\hline Gray screen & & \\
Sway area & $193.799 \pm 85.900$ & $153.259 \pm 76.686$ \\
ML SD & $3.726 \pm 0.892$ & $3.246 \pm 0.865^{*}$ \\
AP SD & $4.292 \pm 1.341$ & $3.951 \pm 1.511$ \\
ML frequency & $0.279 \pm 0.078$ & $0.281 \pm 0.090$ \\
AP frequency & $0.192 \pm 0.071$ & $0.197 \pm 0.094$ \\
ML velocity & $9.290 \pm 1.931$ & $8.351 \pm 1.481^{*}$ \\
AP velocity & $8.656 \pm 1.588$ & $8.147 \pm 1.266$ \\
Neutral & & \\
Sway area & $137.190 \pm 72.152$ & $98.031 \pm 35.223^{*}$ \\
ML SD & $3.366 \pm 1.195$ & $2.864 \pm 0.716$ \\
AP SD & $3.280 \pm 0.798$ & $2.891 \pm 0.666$ \\
ML frequency & $0.301 \pm 0.099$ & $0.284 \pm 0.061$ \\
AP frequency & $0.242 \pm 0.075$ & $0.230 \pm 0.050$ \\
ML velocity & $8.999 \pm 2.177$ & $8.036 \pm 1.430$ \\
AP velocity & $8.553 \pm 1.603$ & $7.930 \pm 1.372$ \\
Anxiogenic & & \\
Sway area & $158.465 \pm 101.369$ & $110.340 \pm 46.547^{*}$ \\
ML SD & $3.960 \pm 1.774$ & $3.156 \pm 0.733^{*}$ \\
AP SD & $3.334 \pm 0.996$ & $2.870 \pm 0.772$ \\
ML frequency & $0.257 \pm 0.094$ & $0.256 \pm 0.059$ \\
AP frequency & $0.246 \pm 0.115$ & $0.254 \pm 0.084$ \\
ML velocity & $9.046 \pm 2.043$ & $7.949 \pm 1.222^{*}$ \\
AP velocity & $8.627 \pm 1.958$ & $7.933 \pm 1.391$ \\
Mutilation & & \\
Sway area & $3.369 \pm 1.060$ & $2.914 \pm 0.844$ \\
ML SD & $3.274 \pm 0.886$ & $2.914 \pm 1.055$ \\
AP SD & $0.308 \pm 0.094$ & $0.298 \pm 0.101$ \\
ML frequency & $0.246 \pm 0.081$ & $0.275 \pm 0.119$ \\
AP frequency & $9.060 \pm 1.910$ & $7.994 \pm 1.487^{*}$ \\
ML velocity & $8.595 \pm 1.584$ & $8.39 \pm 1.538$ \\
AP velocity & & \\
\hline
\end{tabular}

Data are reported as means $\pm S D$. ML SD = standard deviation in the mediolateral direction; AP SD = standard deviation in the anteroposterior direction; ML frequency = frequency of sway in the mediolateral direction; AP frequency = frequency of sway in the anteroposterior direction; $M L$ velocity = mean velocity in the mediolateral direction; $A P$ velocity $=$ mean velocity in the anteroposterior direction. ${ }^{*} \mathrm{P} \leq 0.05 \mathrm{com}-$ pared to control (ANOVA).

\section{Experimental data}

There was no significant difference between groups in sway area during the presentation of the gray screen, but the SAD group exhibited a lower mediolateral SD $(P \leq 0.05)$ and a lower mean velocity in the mediolateral direction $(P \leq 0.05)$ compared to controls during the first gray screen (Table 1).

While viewing neutral pictures, the SAD group exhibited a reduced sway area $(P \leq 0.05)$ compared to the control group and also a lower velocity in the mediolateral direction (Table 1). 


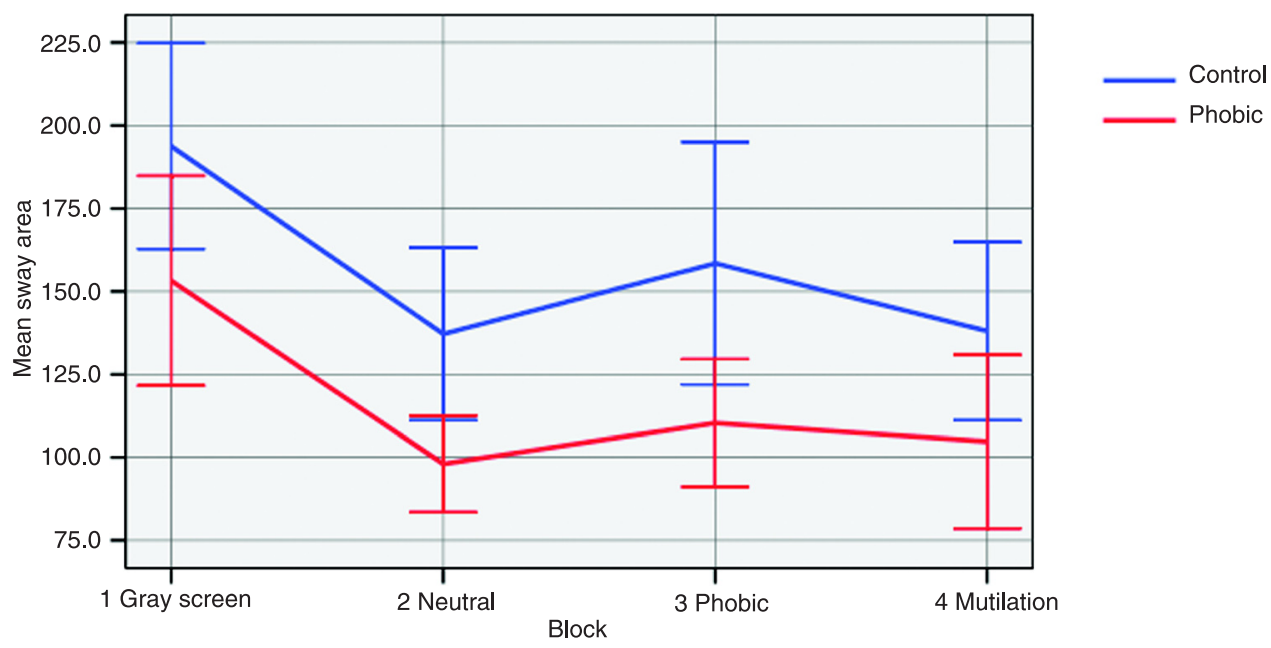

Figure 1. The means $(95 \% \mathrm{Cl})$ of the oscillation tend to stay close, possibly to habituation to the stimuli. In the anxiogenic block, a more specific stimulus, they are apart and then become closer again in the negative block.

While viewing anxiogenic pictures, compared with the control group, the SAD group exhibited a reduced sway area $(P \leq 0.05)$, a reduced mediolateral $S D(P \leq 0.05)$ and a lower velocity in the mediolateral direction $(P \leq 0.05)$ in the anxiogenic block (Table 1).

In the last block of pictures, there was no significant difference between the two groups in sway area in the negative (e.g., images of mutilation) block, but the SAD group exhibited a lower mean velocity in the mediolateral direction $(P \leq 0.05)$ compared to the control group (Table 1).

Figure 1 illustrates the mean sway area of the groups during the images. Initially, the lines tend to stay close, with the mean of the SAD group tending to remain lower during the whole experiment. The greater distance between the lines is found in the phobic block, which reflects the most significant difference between participants in the response to the pictures.

\section{Discussion}

The main findings of our study are that patients with SAD showed a reduced sway area and a lower velocity in the mediolateral direction during presentation of all blocks of pictures compared to control. Contrary to what we hypothesized, the phobic group did not show a significant difference in postural balance when viewing the anxiogenic pictures compared to other types of stimuli, although it was possible to perceive the most significant difference between the means of sway area in this block, which represents a specific stimulus, contrary to the other general images.

Lopes et al. (10) also observed a smaller sway area among subjects with PD compared to healthy controls during all image presentations, except when presenting mutilation pictures. In contrast to Lopes et al. (10), who found an increase in velocity in response to panic stimuli in the anteroposterior direction, we found an increase in the mediolateral direction. We considered the possibility that SAD may have a particular group feature as a "soft sign" that may integrate the factors involved in the differentiation of the anxiety disorders, distinguishing it from PD (2).

The present data show that body sway in patients with SAD is smaller than in controls independently of the presence and contents of visual information. It is possible that the test was not able to provoke extreme anxiety, although the pictures were evaluated by different SAD patients before. Although there was no difference in anxiety between groups before the experiment, SAD subjects ended more anxious, suggesting that they might not have had enough time to habituate to the experiment. Perhaps including one more block before the anxiogenic pictures could help the participants to better assimilate the experiment and a more accurate assessment could be performed.

Our study has some limitations. Since we did not recruit participants free from medication, it is possible that the body oscillation and anxiety were not fully precise as it was expected based on a study in which selective serotonin reuptake inhibitors were shown to reduce postural imbalance in anxious mice (23). Second, we did not use physiological measures, such as skin conductance, which are directly associated with extreme anxiety and may confound the measurement of postural control. We also did not use a randomly mixed design for the presentation of blocks, which could have provided additional results. 


\section{Acknowledgments}

Research supported by CNPq and INCT Translational Medicine (CNPq). Research also partially supported by a fellowship from the Fundação de Apoio ao Ensino, Pes-

\section{References}

1. Maurer $\mathrm{C}$, Mergner T, Xie J, Faist M, Pollak P, Lucking $\mathrm{CH}$. Effect of chronic bilateral subthalamic nucleus (STN) stimulation on postural control in Parkinson's disease. Brain 2003; 126: $1146-1163$

2. Pavol MJ. Detecting and understanding differences in postural sway. Focus on "A new interpretation of spontaneous sway measures based on a simple model of human postural control". J Neurophysiol 2005; 93: 20-21.

3. Brandao ML, Zanoveli JM, Ruiz-Martinez RC, Oliveira LC, Landeira-Fernandez J. Different patterns of freezing behavior organized in the periaqueductal gray of rats: association with different types of anxiety. Behav Brain Res 2008; 188: $1-13$.

4. LeDoux J. The emotional brain. New York: Simon \& Schuster; 1996.

5. Azevedo TM, Volchan E, Imbiriba LA, Rodrigues EC, Oliveira JM, Oliveira LF, et al. A freezing-like posture to pictures of mutilation. Psychophysiology 2005; 42: 255-260.

6. Blanchard RJ, Flannelly KJ, Blanchard DC. Defensive behavior of laboratory and wild Rattus norvegicus. J Comp Psychol 1986; 100: 101-107.

7. Kalin NH. The neurobiology of fear. Sci Am 1993; 268: 94 101.

8. Ishida M. Effect of allocating attention to aversive events on cardiovascular responses and event-related potentials in a dual-task paradigm. Int J Psychophysiol 2006; 62: 93-102.

9. Hüweler R, Kandil FI, Alpers GW, Gerlach AL. The impact of visual flow stimulation on anxiety, dizziness, and body sway in individuals with and without fear of heights. Behav Res Ther 2009; 247: 345-352.

10. Lopes FL, Azevedo TM, Imbiriba LA, Freire RC, Valenca AM, Caldirola D, et al. Freezing reaction in panic disorder patients associated with anticipatory anxiety. Depress Anxiety 2009; 26: 917-921.

11. Galliano G, Noble LM, Travis LA, Puechl C. Victim reactions during rape/sexual assault: a preliminary study of the immobility response and its correlates. $J$ Interpers Violence 1993; 8: 109-114.

12. Lissek S, Levenson J, Biggs AL, Johnson LL, Ameli R, Pine DS, et al. Elevated fear conditioning to socially relevant unconditioned stimuli in social anxiety disorder. Am J Psychiatry 2008; 165: 124-132.

13. Cornwell BR, Johnson L, Berardi L, Grillon C. Anticipation quisa e Assistência do Hospital das Clínicas, Faculdade de Medicina de Ribeirão Preto, Universidade de São Paulo (FAEPA, Brazil). J.A. Crippa (1C), J.E. Hallak (1C) and A.E. Nardi (1A) are recipients of CNPq Productivity fellowship awards. of public speaking in virtual reality reveals a relationship between trait social anxiety and startle reactivity. Biol Psychiatry 2006; 59: 664-666.

14. de Lima Osorio F, Crippa JA, Loureiro SR. A study of the discriminative validity of a screening tool (MINI-SPIN) for social anxiety disorder applied to Brazilian university students. Eur Psychiatry 2007; 22: 239-243.

15. Crippa JA, Loureiro SR, Baptista CA, Osorio F. Are there differences between early- and late-onset social anxiety disorder? Rev Bras Psiquiatr 2007; 29: 195-196.

16. Connor KM, Kobak KA, Churchill LE, Katzelnick D, Davidson JR. Mini-SPIN: A brief screening assessment for generalized social anxiety disorder. Depress Anxiety 2001; 14: 137140.

17. Del-Ben CM, Vilela JAA, Crippa JAS, Hallak JEC, Labate CM, Zuardi AW. Confiabilidade da "Entrevista Clínica Estruturada para o DSM-IV - Versão Clínica" traduzida para o português. Rev Bras Psiquiatr 2001; 23: 156-159.

18. First MB, Spitzer RL, Gibbon M, Williams JBM. Structured Clinical Interview Diagnostic (SCID) for DSM-IV Axis I Disorders - Clinician Version (SCID-CV). Washington: American Psychiatric Press; 1997.

19. Crippa JA, de Lima Osorio F, Del-Ben CM, Filho AS, da Silva Freitas MC, Loureiro SR. Comparability between telephone and face-to-face structured clinical interview for DSM-IV in assessing social anxiety disorder. Perspect Psychiatr Care 2008; 44: 241-247.

20. Bech P, Kastrup M, Rafaelsen OJ. Mini-compendium of rating scales for states of anxiety depression mania schizophrenia with corresponding DSM-III syndromes. Acta Psychiatr Scand Suppl 1986; 326: 1-37.

21. Filho AS, Hetem LA, Ferrari MC, Trzesniak C, Martin-Santos $\mathrm{R}$, Borduqui $\mathrm{T}$, et al. Social anxiety disorder: what are we losing with the current diagnostic criteria? Acta Psychiatr Scand 2010; 121: 216-226.

22. Lang PJ, Bradley MM, Cuthbert BN. International affective picture system (IAPS): affective ratings of pictures and instruction manual. Technical Report A-8. Gainesville: University of Florida; 2008.

23. Venault $P$, Rudrauf $D$, Lepicard EM, Berthoz A, Jouvent R, Chapouthier G. Balance control and posture in anxious mice improved by SSRI treatment. Neuroreport 2001; 12: 30913094. 